\title{
Contact Graphs of Circular Arcs
}

\author{
M. J. Alam ${ }^{1}$, D. Eppstein ${ }^{2}$, M. Kaufmann ${ }^{3}$, S. G. Kobourov ${ }^{1}$, S. Pupyrev ${ }^{1}$, \\ A. Schulz ${ }^{4}$, and T. Ueckerdt ${ }^{5}$ \\ 1 Department of Computer Science, University of Arizona, USA \\ 2 Computer Science Department, University of California, Irvine, USA \\ 3 Wilhelm-Schickard-Institut für Informatik, Universität Tübingen, Germany \\ ${ }^{4}$ Institut Math. Logik \& Grundlagenforschung, Universität Münster, Germany \\ 5 Department of Mathematics, Karlsruhe Institute of Technology, Germany
}

\begin{abstract}
We study representations of graphs by contacts of circular arcs, CCA-representations for short, where the vertices are interiordisjoint circular arcs in the plane and each edge is realized by an endpoint of one arc touching the interior of another. A graph is $(2, k)$-sparse if every $s$-vertex subgraph has at most $2 s-k$ edges, and $(2, k)$-tight if in addition it has exactly $2 n-k$ edges, where $n$ is the number of vertices. Every graph with a CCA-representation is planar and $(2,0)$-sparse, and it follows from known results that for $k \geq 3$ every $(2, k)$-sparse graph has a CCA-representation. Hence the question of CCA-representability is open for $(2, k)$-sparse graphs with $0 \leq k \leq 2$. We partially answer this question by computing CCA-representations for several subclasses of planar (2,0)-sparse graphs. Next, we study CCA-representations in which each arc has an empty convex hull. We show that every plane graph of maximum degree 4 has such a representation, but that finding such a representation for a plane $(2,0)$-tight graph with maximum degree 5 is NP-complete. Finally, we describe a simple algorithm for representing plane $(2,0)$-sparse graphs with wedges, where each vertex is represented with a sequence of two circular arcs (straight-line segments).
\end{abstract}

\section{Introduction}

In a contact representation of a planar graph, the vertices are represented by non-overlapping geometric objects such as circles, polygons, or line segments and the edges are realized by a prespecified type of contact between these objects. Contact graphs of circles, made famous by the Koebe-Andreev-Thurston circle packing theorem 16, have a large number of applications in graph drawing (see e.g. 2 for many references) and this success has motivated the study of many other contact representations $3: 9412$. The special cases of contact representations with curves and line segments are of particular interest [5, 8, 15]. We consider a novel type of contact representation where a vertex is represented by a circular arc, and an edge corresponds to an endpoint of one arc touching an interior point of another arc (Fig. 1). Tangencies between interior points of arcs do not 


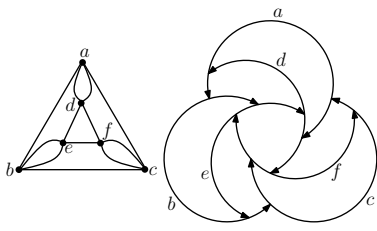

(a)

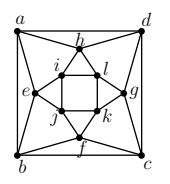

(b)

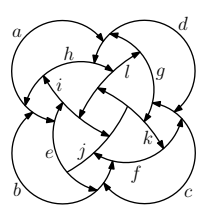

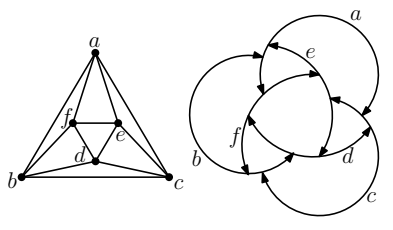

(c)

Fig. 1. CCA representations of a multigraph (a) and two simple graphs (b)-(c).

count as contacts as this would trivialize the problem. These representations are a generalization of the contacts of straight-line segments (which may be thought of as circular arcs with infinite radius) and we call them contacts of circular arcs or CCA-representations for short.

Every $k$-vertex induced subgraph of a contact graph of curves in the plane has at most $2 k$ edges, because every edge uses up one of the curve endpoints. This motivates us to study classes of sparse graphs defined by limits on the numbers of edges in their subgraphs. A graph $G=(V, E)$ is said to be $(p, k)$-sparse [17] if for every $W \subseteq V$ we have $|E[W]| \leq \max \{p|W|-k,|W|-1\}$; it is $(p, k)$-tight if in addition $|E|=p|V|-k$. For example, $(1,1)$-sparse graphs are exactly forests, while $(1,1)$-tight graphs are trees, and the observation above can be rephrased as stating that all graphs representable by circular arcs are $(2,0)$-sparse. This definition makes sense only for $k<2 p$ : for larger $k$, each two vertices would induce no edges and the graph would be empty. However, we may extend the definition by restricting $|W|$ to be larger than two. Thus, we define a graph to be $(2,4)$-sparse if every $s$-vertex subgraph with $s \geq 3$ has at most $2 s-4$ edges, and $(2,4)$-tight if in addition it has exactly $2|V|-4$ edges. A planar graph is $(2,4)$-sparse if and only if it is triangle-free and $(2,4)$-tight if and only if it is a maximal bipartite planar graph. The same idea can be extended to larger $k$ by restricting $|W|$ to be even greater, but in the remainder we consider only planar $(2, k)$-sparse and planar $(2, k)$-tight graphs for $k \in\{0,1,2,3,4\}$.

A graph admits a curve contact representation if and only if it is planar (because the curves do not cross) and (2,0)-sparse (each subset of $s$ curves has at most $2 s$ contacts) [15]. On the other hand, a planar graph has a contact representation with line segments if and only if it is $(2,3)$-sparse [1. Hence, natural questions arise: What are the simplest curves that can represent all planar $(2,0)$-sparse graphs? Perhaps the simplest non-straight curves are circular arcs, so how powerful are circular arcs in terms of contact representations? In particular, does every planar $(2, k)$-sparse graph have a CCA-representation for $k \in\{0,1,2\}$ ? We partially answer these questions by computing circular-arc contact representation for several subclasses of planar $(2,0)$-sparse graphs, and by finding a $(2,0)$-sparse plane multigraph that does not have such a representation.

As another contribution, we resolve an open problem by de Fraysseix and de Mendez [8]. They proved that any contact representation with curves is homeomorphic to one with polylines composed of three segments, and asked if two segments per polyline is sufficient. We affirmatively answer the question. 
Preliminaries. In order to state our results, we need some structural information about sparse planar graphs. We defer the proofs to Appendix A.

\section{Lemma 1 (Augmentations).}

- For every integer $k \in\{0,1,2,3\}$, every plane $(2, k)$-sparse graph is a spanning subgraph of some plane $(2, k)$-tight graph.

- A $(2,4)$-sparse graph forms a subgraph of a $(2,4)$-tight graph if and only if it is bipartite. In particular, the 5-cycle is $(2,4)$-sparse but not a subgraph of a $(2,4)$-tight graph.

\section{Lemma 2 (Plane Duals).}

- For every $k \in\{0,1\}$ and every integer $\ell \in \mathbb{Z}$, there is a plane $(2, k)$-tight graph whose dual is not $(2, \ell)$-sparse.

- For every $k \in\{2,3,4\}$, every plane $(2, k)$-tight graph has a $(2,4-k)$-tight dual.

In particular, duality is an involution on the plane (2,2)-tight graphs, so every plane $(2,2)$-tight graph is the dual of another plane $(2,2)$-tight graph. However, for $k \in\{3,4\}$, the duals of plane $(2, k)$-tight graphs form a proper subclass of all plane $(2,4-k)$-tight graphs. In fact, we prove that the dual of every plane (2,3)-tight graph is a co-Laman graph (a graph where $|E|=2|V|-1$ and $E[W] \leq 2|W|-2$ for all $W \subsetneq V$; see Fekete et al. [11]). The duals of the $(2,4)$-tight graph are exactly the 4-regular plane graphs.

New Results. Our main results are:

\section{Theorem 1 (Contact Circular Arc Representations).}

- Every plane $(2,2)$-sparse graph has a contact representation with circular arcs.

- Every plane co-Laman multigraph has a contact representation with circular arcs.

- Every plane graph with maximum degree 4 has a contact representation with circular arcs.

- There is a plane $(2,0)$-tight multigraph with no contact representation with circular arcs.

The theorem above directly implies the following corollary.

Corollary 1. For every $k \in\{0,1,2,3,4\}$ and every plane $(2, k)$-tight graph $G$, the plane dual $G^{*}$ of $G$ has a contact representation with circular arcs, whenever $G^{*}$ is $(2,4-k)$-tight.

We use two different approaches to construct CCA representations. The first approach is a constructive one, and it can be used for plane $(2,2)$-tight graphs and plane co-Laman graphs. We find a special construction sequence for each graph in these two classes (similar to the Henneberg moves 14 for $(2,3)$-tight graphs, also see $11,21,23$ ), and we show that this construction sequence can be modified into a construction sequence for a CCA-representation. The second approach is structural. For some planar $(2,0)$-sparse graphs, in particular for all graphs 
of maximum degree 4, we can obtain a stronger form of contact representation where the convex hull of each arc is empty. For these graphs we define the notion of a good 2-orientation, and use a circle packing construction to find this stronger CCA-representation from the good orientation. However, we show

Theorem 2. Testing whether a planar $(2,0)$-tight graph has a contact representation where the convex hull of each arc is empty is NP-complete, even for graphs of maximum degree 5 .

Finally we consider contact representation with wedges (that is, polyline segments with at most one bend). A wedge can be viewed as a sequence of two circular arcs (straight-line segments). It is not difficult to prove that every planar $(2,0)$-sparse graph has a contact representation with polylines composed of three segments 8. On the other hand, as pointed out earlier, one segment per polyline is not sufficient. This raises a question (asked in 8 ) whether every planar (2,0)-sparse graph has a contact representation with polylines composed of two segments. We resolve the question by showing that every plane $(2,0)$-sparse graph has a contact representation with wedges.

Theorem 3. Every plane $(2,0)$-sparse graph has a contact representation where each vertex is represented by a wedge.

\section{Contact Representations from Henneberg Moves}

Here we prove the existence of CCA-representations for $(2,2)$-sparse and coLaman graphs, the first two cases of Theorem 11. We defer the degree- 4 and $(2,0)$-tight cases to Section 3

We begin by describing a set of moves which can be applied to a plane $(2, k)$-tight graph, in order to obtain a larger plane $(2, k)$-tight graph (with more vertices), where $k \in\{0,1,2,3,4\}$ depends on the type of move. Afterwards we show that certain subsets of these moves can be used to generate all plane $(2, k)$-tight graphs of a certain class of graphs, starting from one concrete base graph. All but one of these moves are well-known and have already successfully been used for this purpose; see Fig. 2

Definition 1 (Moves). Let $G=(V, E)$ be a plane $(2, k)$-graph for some $k \in$ $\{0,1,2,3,4\}$.

The Henneberg 1 move $H_{1}$. For a face $f$ of $G$ and two distinct vertices $u, v$ on $f$, introduce a new vertex $x$ inside $f$ and add edges from $x$ to $u$ and $v$.

The Henneberg 2 move $\mathrm{H}_{2}$. For a face $f$ of $G$ and an edge $e=(u, v)$ on $f$ and $a$ third vertex $w \neq u, v$ on $f$, introduce a new vertex $x$ inside $f$, add edges from $x$ to $u, v$ and $w$, and remove the edge $e$.

The edge-to- $K_{3}$ move $E_{3}$. For an edge $e=(u, v)$ of $G$ and a face $f$ incident to $v$, replace $v$ by two vertices $v_{1}, v_{2}$ connected by an edge $\left(v_{1}, v_{2}\right)$, and add edges from $v_{1}\left(v_{2}\right)$ to each neighbor of $v$ that lies clockwise (counterclockwise) between $f$ and e (included) around $v$. 


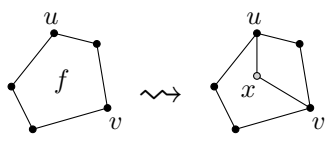

Henneberg 1 move $H_{1}$

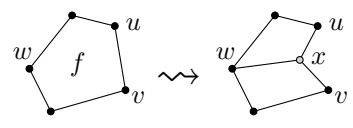

Henneberg 2 move $\mathrm{H}_{2}$

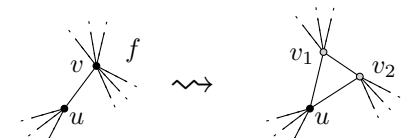

edge-to- $K_{3}$ move $\dot{E}_{3}$

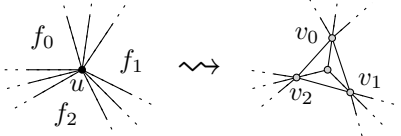

vertex-to- $K_{4}$ move $V_{4}$

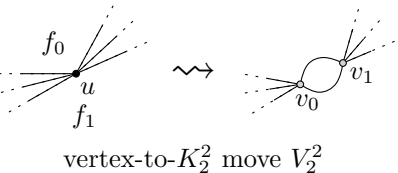

vertex-to- $K_{2}^{2}$ move $V_{2}^{2}$

Fig. 2. The moves $H_{1}, H_{2}, E_{3}, V_{4}$ and $V_{2}^{2}$.

The vertex-to- $K_{4}$ move $V_{4}$. For a vertex $u$ of $G$ and three (not necessarily distinct) faces $f_{0}, f_{1}, f_{2}$ incident to $u$, appearing in that clockwise order around $u$, replace $u$ by a plane $K_{4}$ with outer vertices $v_{0}, v_{1}, v_{2}$, and add edges from $v_{i}$ to every neighbor of $u$ that lies clockwise between $f_{i}$ and $f_{i+1}$ around $u$, $i=0,1,2$, where indices are taken modulo 3 .

The vertex-to- $K_{2}^{2}$ move $V_{2}^{2}$. For a vertex $u$ of $G$ and two (not necessarily distinct) faces $f_{0}, f_{1}$ at $u$, replace $u$ by two vertices $v_{1}, v_{2}$ connected by two parallel edges, and add edges from $v_{i}$ to every neighbor of $u$ that lies clockwise between $f_{i}$ and $f_{i+1}$ around $u, i=0,1$, where indices are taken modulo 2 .

Henneberg moves $H_{1}$ and $H_{2}$ were introduced by Henneberg [14, moves $E_{3}$ and $V_{2}^{2}$ were defined by Whiteley [23], $E_{3}$ also appears in [11] under the name vertex-splitting, and the move $V_{4}$ was introduced by Nixon and Owen 21.

Part (i) of Lemma 3 is due to Henneberg [14, see also Haas et al. 13.

Lemma 3. Each of the following holds.

(i) All plane $(2,3)$-tight graphs can be generated by $\mathrm{H}_{1}$ and $\mathrm{H}_{2}$ moves starting from a triangle [13,14].

(ii) All duals of plane $(2,3)$-tight graphs can be generated by $E_{3}$ and $V_{2}^{2}$ moves starting from three parallel edges.

(iii) All plane $(2,2)$-tight graphs can be generated by $E_{3}$ and $V_{4}$ moves starting from an isolated vertex.

In order to prove (iii) we need one more concept from the literature. A Lamanplus-one graph is a simple graph $G$ with an edge $e=u v$, so that $\operatorname{deg}(u) \geq 2$ and $\operatorname{deg}(v) \geq 2$, and $G-e$ is a $(2,3)$-tight (Laman) graph. Laman-plus-one graphs form a proper subclass of $(2,2)$-tight graphs. Fekete et al. [11] claimed the following without a proof on generating Laman-plus-one graphs by $E_{3}$ moves starting from $K_{4}$. For the sake of completeness we prove for the claim in Appendix B.

Lemma 4 (Fekete et al. [11]). Every plane Laman-plus-one graph can be generated by $E_{3}$ moves starting from $K_{4}$.

For $(2,2)$-tight graphs that are not Laman-plus-one, Nixon [20] proved: 
Lemma 5 (Nixon [20]). Let $G$ be a $(2,2)$-tight graph with at least one edge. Then $G$ is a Laman-plus-one graph or there exists a proper $(2,2)$-tight subgraph $H$ of $G$ such that no vertex of $G-H$ is adjacent to more than one vertex in $H$.

For a subgraph $H$ of a graph $G$, let $V(H)$ and $E(H)$ be the vertex set and the edge set of $H$. Denote by $G-H$ the subgraph of $G$ induced by the vertices $V(G) \backslash V(H)$. For two subgraphs $H_{1}$ and $H_{2}$ of $G$, let $H_{1} \cup H_{2}$ be the subgraph $H=\left(V\left(H_{1}\right) \cup V\left(H_{2}\right), E\left(H_{1}\right) \cup E\left(H_{2}\right)\right)$. Let $E\left(H_{1}, H_{2}\right)$ be the set of edges between the vertices of $H_{1}$ and $H_{2}$. The proof of the following Lemma is in Appendix B

Lemma 6. Let $G$ be a $(2, k)$-tight graph and let $H$ be a proper $(2, k)$-tight subgraph of $G$, for $k>0$. Let $C$ and $D$ be a partition of the vertices of $G-H$ such that there is no edge between vertices in $C$ and vertices in $D$. Then both the graphs induced by the vertices of $H \cup C$ and $H \cup D$ are also $(2, k)$-tight.

We are now ready to prove Lemma 3

Proof.[Proof of Lemma 3 Part (i1), that every plane Laman graph can be generated by $H_{1}$ and $H_{2}$ moves starting from a triangle, is already known [13.14.

Now, if $G^{*}$ is the plane dual of a plane Laman graph $G$, then we follow the construction sequence of $G$ with $H_{1}$ and $H_{2}$ moves in the dual and observe that this gives a construction sequence of $G^{*}$ with $V_{2}^{2}$ and $E_{3}$ moves, starting with three parallel edges. This proves (ii).

Let $G$ be a plane $(2,2)$-tight graph. We prove by induction on $|V(G)|$, that $G$ can be generated by $E_{3}$ and $V_{4}$ moves, starting with a vertex. If $|V(G)|=1$, this clearly holds. Assume that $|V(G)| \geq 2$. If $G$ is Laman-plus-one, it can be obtained from a single vertex by a single $V_{4}$ move, followed by a number of $E_{3}$ moves, by Lemma 4 and the claim follows. Otherwise, by Lemma 5 , there exists a proper (2,2)-tight subgraph $H$ of $G$ such that no vertex of $G-H$ is adjacent to more than one vertex in $H$. Furthermore, since $G$ is $(2,2)$-tight, $H$ is connected. Since $H$ is a proper subgraph of $G$, assume without loss of generality that the outer face of $H$ is not vertex-empty in $G$ (otherwise at least one internal face is not vertex-empty and a similar reasoning holds). Let $H^{\prime}$ be the subgraph of $G$ consisting of $H$ and all the vertices inside the outer boundary of $H$. By Lemma 6. $H^{\prime}$ is also a planar $(2,2)$-tight graph, which is a proper subgraph of $G$. Thus by the induction hypothesis, $H^{\prime}$ can be constructed from a single vertex by $E_{3}$ and $V_{4}$ moves. Let $\Pi_{1}$ denote this sequence of these two moves. The graph $G^{\prime}$ obtained from $G$ by merging $H^{\prime}$ into a single vertex is simple and planar $(2,2)$-tight. By the induction hypothesis, $G^{\prime}$ can be obtained from a single vertex by a sequence $\Pi_{2}$ of $E_{3}$ and $V_{4}$ moves. The sequence $\Pi_{2}$ followed by the sequence $\Pi_{1}$ generates $G$ from a vertex.

Lemma 7. Let $G$ be a plane graph with a $C C A$-representation and $G^{\prime}$ be a plane graph obtained from $G$ by $a V_{4}, E_{3}$ or $V_{2}^{2}$ move. Then $G^{\prime}$ admits a $C C A$ representation as well.

Proof. Let $G^{\prime}$ be obtained from $G$ by a $V_{4}, E_{3}$ or $V_{2}^{2}$ move. In each case, we show how to locally modify a given CCA-representation of $G$ into a CCA-representation 

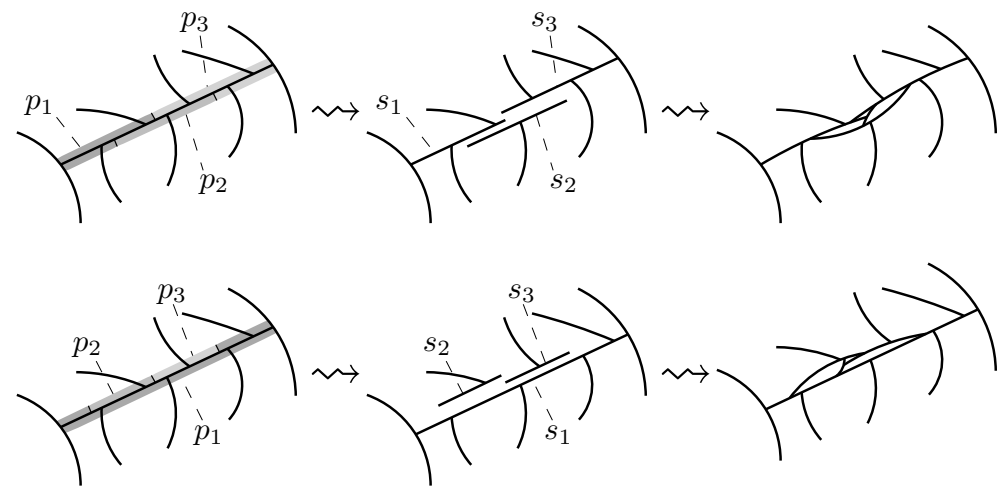

Fig. 3. Performing a $V_{4}$ move in a CCA-representation.

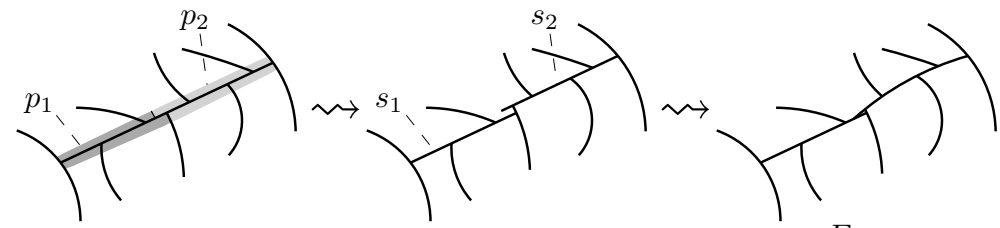

$E_{3}$ move
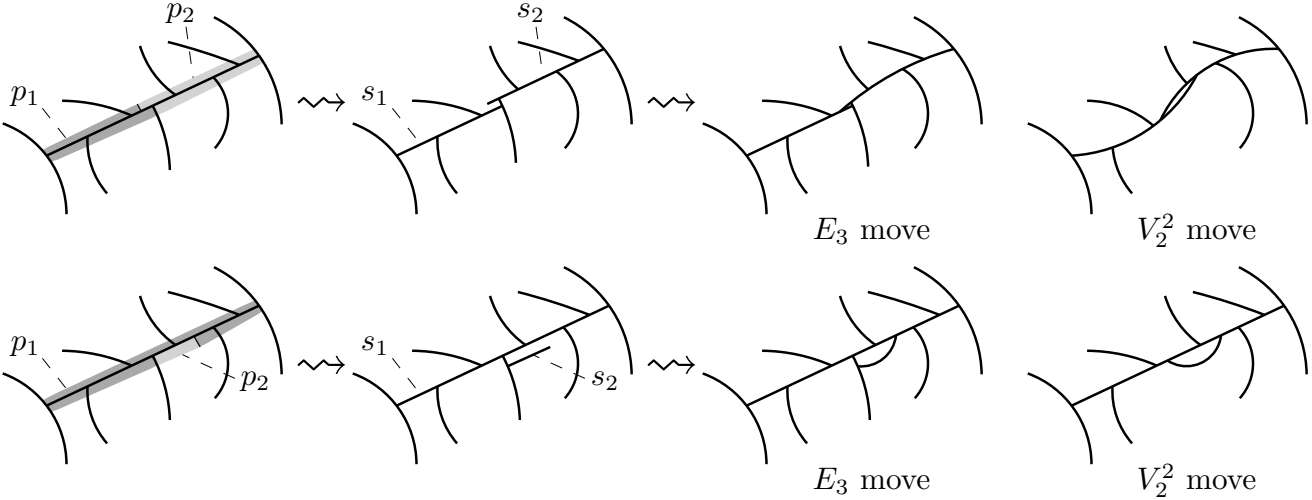

Fig. 4. Performing an $E_{3}$ move and $V_{2}^{2}$ move in a CCA-representation.

of $G^{\prime}$. All the cases are similar, so we restrict ourselves to a careful description of the first case only, and provide figures illustrating the remaining cases.

Let $v$ be the vertex in $G$ and $\left\{v_{1}, v_{2}, v_{3}, v_{4}\right\}$ be the four vertices in $G^{\prime}$ that replace $v$. Let $S_{i}=N_{G^{\prime}}\left(v_{i}\right) \backslash\left\{v_{1}, v_{2}, v_{3}, v_{4}\right\}, i=1,2,3,4$. By definition, we have $S_{i} \cap S_{j}=\emptyset$ for $i \neq j, S_{4}=\emptyset, S_{1} \cup S_{2} \cup S_{3}=N_{G}(v)$ and each of $S_{1}, S_{2}, S_{3}$ forms a subset of $N_{G}(v)$ that appears consecutively in the clockwise order around $v$ in $G$. Assume without loss of generality that the circular arc $c_{v}$ for $v$ in the given CCA-representation of $G$ is a straight segment. The boundary of $c_{v}$ can be partitioned into three consecutive pieces $p_{1}, p_{2}, p_{3}$, so that $p_{i}$ contains exactly the contacts corresponding to vertices in $S_{i}, i=1,2,3$; see Fig. 3 .

From the pieces $p_{1}, p_{2}, p_{3}$, we define straight segments $s_{1}, s_{2}, s_{3}$ parallel to $c_{v}$ so that each $s_{i}$ intersects exactly the circular arcs for vertices in $S_{i}, i=1,2,3$. Then, each $s_{i}$ is "curved" into a circular arc, so that $s_{1}, s_{2}, s_{3}$ form a triangle with one free endpoint on the inside. We add a fourth circular arc for $v_{4}$ in the triangle, containing the free endpoint in its interior and touching the other two circular arcs with its two endpoints; see Fig. 3 . 


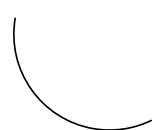

(a)

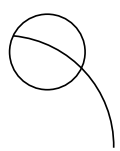

(b)

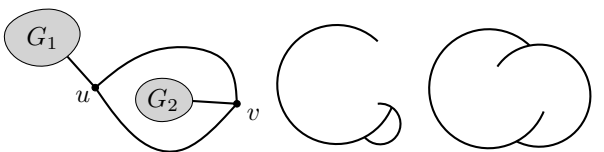

(c)

Fig. 5. The base case $G_{0}$ for (a) (2,2)-tight graphs and (b) co-Laman graphs. (c) A plane $(2,0)$-tight graph that does not admit a CCA-representation.

The cases for $E_{3}$ move or $V_{2}^{2}$ are similar; the only difference is that we define only two sets $S_{1}, S_{2}$ (with $S_{1} \cap S_{2}=\{u\}$ for an $E_{3}$ move) and consequently only two pieces $p_{1}, p_{2}$ and two straight segments $s_{1}, s_{2}$; see Fig. 4 .

Finally, we prove the main theorem of this section.

Proof.[Proof of Theorem 1] Cases 1 and 2] Let $G$ be a plane graph. We show that $G$ admits a CCA-representation, provided it is $(2,2)$-sparse or a co-Laman graph.

Case 1, $G$ is $(2,2)$-tight. By Lemma $3, G$ can be generated by $E_{3}$ and $V_{4}$ moves, starting from a graph $G_{0}$ with a single vertex. Since $G_{0}$ admits a CCArepresentation (Fig. 5(a) , by Lemma 7, $G$ also has a CCA-representation.

Case 2, $G$ is co-Laman. By Lemma 3, $G$ can be generated by $E_{3}$ and $V_{2}^{2}$ moves, starting from a graph $G_{0}$ with two vertices and three edges. Since $G_{0}$ has a CCA-representation (Fig. [5(b)], by Lemma 7, so does $G$.

\section{Good Orientations and One-Sided Representations}

In order to prove Theorem 1 we need an additional concept of a plane graph $G$.

An orientation of a graph $G$ is a directed graph whose underlying undirected graph is $G$. We call it a 2-orientation if every vertex has out-degree exactly 2, and a $2^{-}$-orientation if every vertex has out-degree at most 2 . An orientation of $G$ is called good if, for every vertex $v$ of $G$, all the outgoing edges (equivalently incoming edges) incident to $v$ are consecutive in the circular ordering of the edges around $v$. A CCA-representation is called one-sided if, for each $\operatorname{arc} a$, the endpoints of other arcs that touch $a$ all do so on one side of $a$. This analogous to the concept of one-sided segment contact representations [10,15]. A CCArepresentation is interior-disjoint if each arc has nonzero curvature and the interior of the convex hull of each arc is disjoint from all the other arcs.

Lemma 8. A simple plane graph with a good $2^{-}$-orientation has an interiordisjoint CCA-representation.

Proof. Consider a plane graph $G$ with a good $2^{-}$-orientation. As with any plane graph, $G$ has a contact representation with disks 16. For each vertex $v$ of $G$ with out-deg $(v)=2$, the two outgoing edges of $v$ define two points, $p$ and $p^{\prime}$, on the circle $C(v)$ representing $v$. If out-deg $(v)=1$, the outgoing edge defines $p$ and we choose $p^{\prime} \in C(v)$ very close to it. If out-deg $(v)=0$ we choose $p$ and 


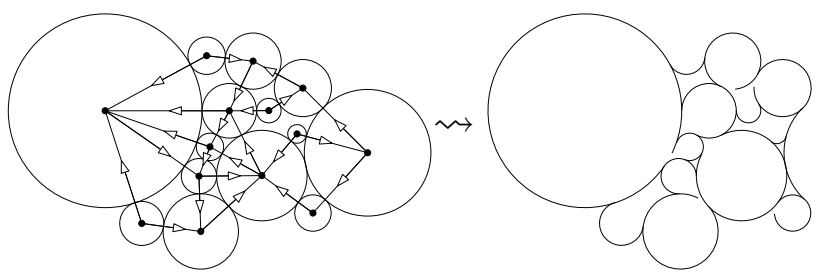

(a)

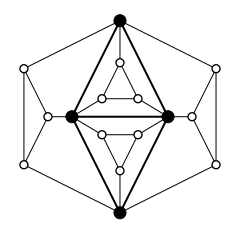

(b)

Fig. 6. (a) From a contact representation with disks and a good $2^{-}$-orientation to a CCA-representation. (b) A planar Laman graph with the minimum degree 3, that has no good 2-orientation.

$p^{\prime}$ distinct from all contacts of $C(v)$ and close to each other. In both cases the two points, $p$ and $p^{\prime}$, split $C(v)$ into two circular arcs. Since the $2^{-}$-orientation is good, one of these two arcs contains none of the contacts of $C(v)$ with other disks. We represent each vertex $v$ by the other circular arc defined by $C(v), p$ and $p^{\prime}$, which contains all the contacts of $C(v)$; see Fig. 6(a).

Lemma 9. Every plane graph $G$ with maximum degree 4 has a good $2^{-}$-orientation.

Proof. First note that vertices of degree strictly less than 4 are harmless, as long as they have at most two outgoing edges: their outgoing edges (if any) cannot be non-consecutive. In order to find a $2^{-}$-orientation of $G$ in which every vertex of degree 4 has consecutive outgoing edges, we define a number of walks in $G$. Start with any edge and define a walk so that, when entering some vertex $v$ of degree 4 via edge $e$ the walk always continues with the edge $e^{\prime}$ that lies opposite of $e$ at $v$. At a degree- 3 vertex that has not already been made part of one of these walks, continue the walk with an arbitrary incident edge, and otherwise stop. Orienting every edge in this walk consistently and starting another iteration with any so-far unoriented edge (if any exists), eventually gives a good $2^{-}$-orientation of $G$.

Not every planar $(2,0)$-sparse graph has a good orientation; a counterexample is easy to construct by adding sufficiently many degree- 2 vertices. Moreover, there is a counterexample with minimum degree 3; see Fig. 6(b). Indeed, the bold subgraph (induced by the black vertices) has five edges and four vertices. Thus, in any $2^{-}$-orientation, at least one black vertex has two bold outgoing edges. At this vertex, all light edges are incoming, breaking up its outgoing edges.

Lemma 10. For a simple plane graph $G$, the following statements are equivalent.

(1) $G$ has a good $2^{-}$-orientation

(2) $G$ has a one-sided CCA-representation

(3) $G$ has an interior-disjoint CCA-representation

Proof. The implication (3) $\Rightarrow(2)$ is obvious (every interior-disjoint representation is one-sided) and the $(1) \Rightarrow(3)$ is Lemma 8 . It remains to prove that every graph with a one-sided CCA-representation has a good $2^{-}$-orientation. But each vertex of the $2^{-}$-orientation derived from a CCA-representation has incoming edges on 
the two sides of the corresponding arc separated by outgoing edges at the two arc endpoints; in a one-sided representation, one set of incoming edges is empty and cannot separate the outgoing edges.

Proof.[Proof of Theorem 11. Cases 3, 4] Let $G$ be a plane graph with the maximum degree 4 . By Lemma 9 , $G$ admits a good $2^{-}$-orientation; and hence by Lemma 8 $G$ admits a CCA-representation. This completes Case 3.

A plane $(2,0)$-tight multigraph with no CCA-representation is shown in Fig. 5(c). It has two vertices $u, v$ joined by two parallel edges $e, e^{\prime}$, and two plane $(2,0)$-tight subgraphs $G_{1}$ and $G_{2} . G_{1}$ lies in the unbounded region and $G_{2}$ lies in the bounded region defined by $e, e^{\prime}$, and $u$ and $v$ are connected by an edge to a vertex in $G_{1}$ and $G_{2}$, respectively. $G$ is plane and $(2,0)$-tight and admits no CCA-representation since two touching circular arcs have their free ends either both in the bounded or both in the unbounded region defined by the closed created curve (Fig. 5(c)) . Note that whether every planar $(2,0)$-tight multigraph has a plane embedding that has a CCA-representation is an open question.

As noted in the introduction, Case 3 of the proof of Theorem 1 always constructs an interior-disjoint CCA-representation for graphs of maximum degree 4 . We now show that finding such representations without the degree constraint is hard. We only provide a sketch here; the details are in Appendix C.

Proof Sketch.[Theorem 2 It is sufficient to prove that finding a good representation of a $(2,0)$-tight graph is NP-complete. We first prove this for plane multigraphs. We reduce from the known NPcomplete problem Positive Planar 1-in-3SAT [18. Our reduction uses wire, splitter, and clause gadgets (Fig. 7) based on the fact that, for a good 2-orientation at a degree4 vertex, each incoming edge is opposite to an outgoing edge and vice versa. Each variable of the 3SAT formula is a wire gadget, that is closed to a circle with dou-

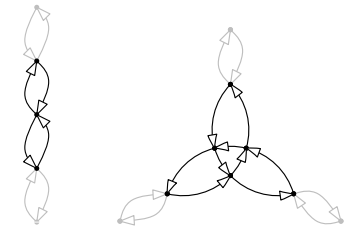

(a) wire (b) splitter

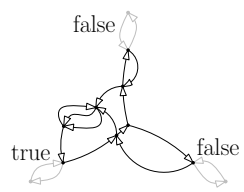

(c) clause

Fig. 7. The gadgets used in the reduction; gray edges show how adjacent gadgets are connected.

2-orientations of this circle, encod-

ing the truth value of the variable. A splitter gadget with a short piece of wire propagate this signal to the clause gadgets, representing clauses. Due to the degree- 4 vertices, there is only one good 2-orientation for the splitter that extends a "wire signal". The degree-3 vertex in the clause gadget verifies that exactly two of the attached wires carry a false signal. Finally we convert this multigraph to a $(2,0)$-tight simple planar graph, keeping the existence of a good 2-orientation; see Appendix C for details. 


\section{Contact Representations with Wedges}

A wedge is a polyline segment with at most one bend (thus two circular arcs). Here we show that any plane $(2,0)$-sparse graph admits a contact representation with wedges. Appendix D considers representation with constrained wedges.

Theorem 3. Every plane (2,0)-sparse graph has a contact representation where each vertex is represented by a wedge.

Proof. A plane (2,0)-sparse graph $G$ has a $2^{-}$-orientation [4, 6, 19]. Consider a straight-line drawing of $G$. For each vertex $v$, the wedge for $v$ is the union of the line segments representing the outgoing edges from $v$. Here all the contacts representing the incoming edges for a vertex is at the bend-point of the wedge, but a small perturbation of the representation is

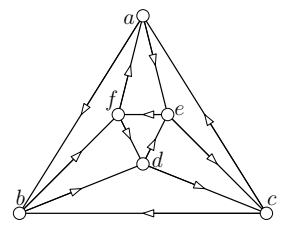

(a)

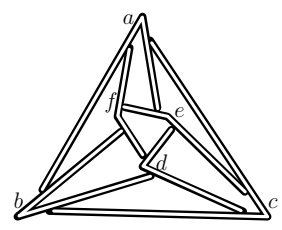

(b)

Fig. 8. (a) A straight-line drawing and a 2-orientation of a $(2,0)$-sparse graph $G$, (b) a contact representation of $G$ with wedges. sufficient to get rid of this degeneracy; see Fig. 8. Indeed, one can slide the endpoint of every wedge a bit along the wedge it is touching, taking care that the endpoints of wedges with smaller incoming angle slide a little further.

\section{Conclusion and Open Questions}

We presented new results about contact representations of graphs with circular arcs. Although every graph with such a contact representation is planar and $(2,0)$-sparse, we provided a $(2,0)$-tight plane multigraph that does not admit such a representation. On the other hand, we identified several subclasses of plane $(2,0)$-sparse graphs that have CCA representations. The natural question remains open: does every simple planar $(2,0)$-sparse graph have a circular-arc contact representation, if we allow changing the embedding?

A circular-arc contact representation $\Gamma$ for a $(2,0)$-tight graph $G$ defines a 3-regular skeleton graph 22], where the points of contact are vertices and arcs between contacts are edges (Fig. 1). Each vertex of $G$ with degree $d \geq 3$ corresponds to a path of $d-2$ vertices in $\Gamma$ along one circular arc. Thus one way to find a circular-arc contact representation for a plane $(2,0)$-tight graph $G$ is to find a 3-regular graph by splitting each vertex of degree $d>3$ into $(d-2)$ degree-3 vertices (each choice of splitting corresponds to a different $2^{-}$-orientation), and align the path associated with each vertex of $G$ into a circular arc. Given a 3 -regular graph with a path-cover, to find a representation with each path aligned as a circular arc is related to the stretchability question $[7$, which is still open.

We also showed that every plane $(2,0)$-sparse graph has a contact representation with polyline segments with a single bend (wedges). In this context, several questions seem interesting: does every $(2,0)$-sparse graph admit a contact representation with equilateral wedges (i.e., wedges with equal-length segments)? Can we bound the smallest angle at the corner of the wedges (to say, $\left.45^{\circ}\right)$ ? 


\section{References}

1. J. Alam, T. Biedl, S. Felsner, M. Kaufmann, and S. Kobourov. Proportional contact representations of planar graphs. J. Graph Algor. Appl., 16(3):701-728, 2012.

2. M. J. Alam, D. Eppstein, M. T. Goodrich, S. G. Kobourov, and S. Pupyrev. Balanced circle packings for planar graphs. In GD 2014, volume 8871 of LNCS, pages 125-136. Springer, 2014.

3. M. Badent, C. Binucci, E. Di Giacomo, W. Didimo, S. Felsner, F. Giordano, J. Kratochvíl, P. Palladino, M. Patrignani, and F. Trotta. Homothetic triangle contact representations of planar graphs. In CCCG 2007, pages 233-236, 2007.

4. O. Bernardi and É. Fusy. A bijection for triangulations, quadrangulations, pentagulations, etc. J. Combinatorial Th., Ser. A, 119(1):218-244, 2012.

5. N. de Castro, F. Cobos, J. Dana, A. Márquez, and M. Noy. Triangle-free planar graphs and segment intersection graphs. J. Graph Algor. Appl., 6(1):7-26, 2002.

6. H. de Fraysseix and P. Ossona de Mendez. On topological aspects of orientations. Discrete Math., 229(1-3):57-72, 2001.

7. H. de Fraysseix and P. Ossona de Mendez. Barycentric systems and stretchability. Discrete Applied Math., 155(9):1079-1095, 2007.

8. H. de Fraysseix and P. Ossona de Mendez. Representations by contact and intersection of segments. Algorithmica, 47(4):453-463, 2007.

9. C. A. Duncan, E. R. Gansner, Y. Hu, M. Kaufmann, and S. G. Kobourov. Optimal polygonal representation of planar graphs. Algorithmica, 63(3):672-691, 2012.

10. D. Eppstein, E. Mumford, B. Speckmann, and K. Verbeek. Area-universal and constrained rectangular layouts. SIAM J. Comput., 41(3):537-564, 2012.

11. Z. Fekete, T. Jordán, and W. Whiteley. An inductive construction for plane Laman graphs via vertex splitting. In ESA 2004, LNCS, pages 299-310. Springer, 2004.

12. D. Gonçalves, B. Lévêque, and A. Pinlou. Triangle contact representations and duality. Discrete Comput. Geom., 48(1):239-254, 2012.

13. R. Haas, D. Orden, G. Rote, F. Santos, B. Servatius, H. Servatius, D. L. Souvaine, I. Streinu, and W. Whiteley. Planar minimally rigid graphs and pseudotriangulations. Comput. Geom. Th. Appl., 31(1-2):31-61, 2005.

14. L. Henneberg. Die graphische Statik der starren Systeme. BG Teubner, 1911.

15. P. Hliněný. Classes and recognition of curve contact graphs. J. Combinatorial Th., Ser. B, 74(1):87-103, 1998.

16. P. Koebe. Kontaktprobleme der konformen Abbildung. Ber. Sächs. Akad., Math.Phys. Klasse, 88:141-164, 1936.

17. A. Lee and I. Streinu. Pebble game algorithms and sparse graphs. Discrete Math., 308(8):1425-1437, 2008.

18. W. Mulzer and G. Rote. Minimum-weight triangulation is NP-hard. J. $A C M, 55(2)$, 2008.

19. C. S. J. A. Nash-Williams. Edge-disjoint spanning trees of finite graphs. J. London Math. Soc., 36:445-450, 1961.

20. A. Nixon. Rigidity on Surfaces. PhD thesis, Lancaster University, 2011.

21. A. Nixon and J. Owen. An inductive construction of $(2,1)$-tight graphs. Unpublished preprint, 2011.

22. A. Schulz. Drawing graphs with few arcs. In $W G 2013$, volume 8165 of $L N C S$, pages 406-417. Springer, 2013.

23. W. Whiteley. Some matroids from discrete applied geometry. In Matroid Theory, volume 197 of Contemp. Math., pages 171-311. Amer. Math. Soc., 1996. 


\section{A Structural Results on Sparse Planar Graphs}

Here we give some preliminary results on $(2, k)$-tight graphs and prove Lemmas 1 and 2

\section{A.1 Spanning Trees and Orientations}

We start by listing some known characterizations of $(2, k)$-tight graphs for $k=2,3$.

Lemma 11. A graph $G$ is $(2,2)$-tight if and only if $G$ is the union of two edgedisjoint spanning trees (Tay [26]; see also [19,20]). $G$ is $(2,3)$-tight if and only if for every edge $e$ of $G$ the graph obtained by doubling e is $(2,2)$-tight. (Graver et al. [24])

Lemma 12 characterizes $(2, i)$-tight graphs in terms of their orientations.

Lemma 12. Let $G=(V, E)$ be a graph. Then each of the following holds.

(i) $G$ is $(2,0)$-tight if and only if $G$ admits a 2-orientation.

(ii) $G$ is $(2,1)$-tight if and only if $G$ plus a loop at an arbitrary vertex $v \in V$ admits a 2-orientation in which for every $u \in V$ there exists a directed $u$-to-v path.

(iii) $G$ is $(2,2)$-tight if and only if $G$ after adding two loops at an arbitrary vertex $v \in V$ has a 2-orientation where for every $u \in V$ there are two edge-disjoint directed $u-$ to-v paths.

(iv) If $G$ is $(2, k)$-sparse for some $k \geq 0$, then $G$ admits a $2^{-}$-orientation.

Proof. We prove this lemma, following the pebbling game of Lee and Streinu [17, according to which a graph is $(2, k)$-sparse (for $0 \leq k<4$ ) if and only if it is possible to construct it, starting from a graph with no edges and two pebbles per vertex, by a sequence of the following operations:

(O1) Add an edge between two vertices that together have at least $k+1$ pebbles while no vertex has more than two pebbles. Remove one of the pebbles and direct the edge away from the vertex from which it was removed.

(O2) Move a pebble backwards along a directed path in the graph, to a vertex that did not already have two pebbles, reversing the orientation of all the edges it moves along.

An easy induction shows that at each step of this process each vertex with $i$ pebbles on it has exactly $2-i$ outgoing edges. Note that case (iv) of the lemma follows immediately, since every $(2, k)$-sparse graph is also $(2,0)$-sparse. We now prove for the remaining three cases.

For case (ii), observe that each operation (O1) uses up one of the initial $2|V|$ pebbles, so a $(2, k)$-tight graph must have exactly $k$ pebbles remaining at the end of the process. In particular a $(2,0)$-tight graph has no pebbles and each vertex has exactly 2 outgoing edges.

For case iii, the oriented graph resulting from the pebbling game will have a pebble on exactly one vertex $v$. There can be no subset of vertices that does 
not contain $v$ and has no outgoing edges, because such a subset would induce a $(2,0)$-tight subgraph, so every vertex has a path to $v$. Adding a self-loop at $v$ gives the desired structure. The vertex $v$ can be chosen arbitrarily, because the pebble can be moved backwards along the path that connects any other vertex to $v$.

For case iii the pebbling game leaves two pebbles on vertices. There can be no subset of vertices for which the number of pebbles in the subset plus the number of edges escaping the subset is less than two, because such a subset would induce a $(2,0)$-tight or $(2,1)$-tight subgraph. Therefore, by similar reasoning to case ii these two pebbles can both be moved to an arbitrarily chosen vertex $v$, after which every other vertex must have two edge-disjoint oriented paths to $v$.

\section{A.2 Augmenting Sparse Graphs to Tight Graphs}

In this section we prove Lemma 1 , that for $k \in\{0,1,2,3\}$ every plane $(2, k)$-sparse graph can be augmented, by adding edges, into a plane $(2, k)$-tight graph. For $k=4$ such an augmentation exists if and only if the graph is bipartite. We distinguish the five cases for $k$.

Case $1, k=0$. If we allow multigraphs, then it is easy to augment $G$. By Lemma 12, $G$ has an orientation with out-deg $(v) \leq 2$ for each $v \in V$. If $\operatorname{out}-\operatorname{deg}(v)=2$ for each $v \in V, G$ is already $(2,0)$-tight. Otherwise, take a vertex $v$ with out-deg $(v) \leq 1$ and double the outgoing edge incident to $v$ to increase the out-degree without breaking planarity.

We prove the stronger claim that any plane $(2,0)$-sparse simple graph with $n \geq 6$ vertices can be augmented to a plane $(2,0)$-tight simple graph. Assume for a contradiction that there is a plane $(2,0)$-sparse simple graph with $n \geq 6$ vertices that cannot be augmented in this way, and let $G$ be such a graph with the maximum number of edges. Since $G$ is not $(2,0)$-tight and $n \geq 6, G$ has a non-triangular face. If $G$ has no $(2,0)$-tight subgraph, then we can add an edge inside such a face without violating $(2,0)$-sparseness and planarity, contradicting the maximality of $G$. Otherwise, there is an inclusion-maximal $(2,0)$-tight subgraph $H$ of $G$. Choose an edge $e=(u, v)$ in $H$ and a vertex $w$ not in $H$, on the same face of $G$. Since $H$ is inclusion-maximal, at least one of the edges $(u, w)$ and $(v, w)$ is not in $G$ and can be added, again contradicting the maximality of $G$.

Case 2, $k=1$. We play the pebble game of Lee and Streinu [17] for the $(2,1)$ sparse graph and end with a directed graph $D=(V, \bar{E})$ whose vertices might contain pebbles. Let $x \in V$ be a vertex with a pebble and let $V_{x}$ be the set of vertices that have a path to $x$ in $D$. If two sets $V_{x}$ and $V_{y}$ intersect (with $x \neq y$ ) we can move two pebbles to the endpoint of an edge and then add a copy of this edge while following the pebble game rules, giving a valid augmentation of the graph. Otherwise, for each $x \neq y$, the sets $V_{x}$ and $V_{y}$ are disjoint. We can further assume that there is no edge between two different vertex sets $V_{x}$ and $V_{y}$ since this edge would extend one of the sets. 
Now let $\bar{V}$ denote all vertices that are in none of the sets $V_{x}$. We have to rule out the case that $D$ restricted to $\bar{V}$ "separates" the sets $V_{x}$. Assume that $\bar{V} \neq \emptyset$. Let $e$ be the edge last added with both endpoints in $\bar{V}$. To add $e$, we needed two pebbles. One pebble remained at some vertex $u \in \bar{V}$ after the addition of $e$. Since $\bar{V}$ has no pebbles, this pebble must have been removed from $\bar{V}$, leaving a path that connects $u$ to the vertex where the pebble remains, or where it was consumed. This place lies outside of $\bar{V}$, say in $V_{x}$. This shows that $u \in V_{x}$, a contradiction.

The augmentation can now be carried out as follows. The graph $D$ decomposes into connected components, associated with the sets $V_{x}$. Select two components, move the free pebbles to the boundaries, and add an edge that connects the two pebbled vertices.

Case 3, $k=2$. By Lemma 11, a graph is $(2,2)$-tight if and only if its edges can be partitioned into two spanning trees. A partition of the edges into two forests $F_{1}$ and $F_{2}$ can be obtained by several known algorithms, [25] for example. Next find a face $f$ and two vertices $u, v$ on $f$ that are not in the same component of one of the forests, say $F_{1}$. If $f$ has at least two independent edges in $F_{2}$, one can add an edge into it and put this edge into $F_{1}$ so that two components of $F_{1}$ merge. Otherwise exactly two edges of $f$ are in $F_{2}$, both incident to the same vertex. Now we consider the faces on the other side of the edge in $F_{2}$ and repeat the argument until we find a face with two independent edges in $F_{2}$ this way. We find such a face at some point; since otherwise we have a cycle of faces with all edges on the outer boundary in $F_{1}$, which gives a cycle in $F_{1}$, a contradiction. Repeated insertion of edges in this way eventually turns both $F_{1}$ and $F_{2}$ into trees and hence $G$ becomes $(2,2)$-tight.

Case $4, k=3$. If $G=(V, E)$ is $(2,3)$-sparse but has fewer than $2|V|-3$ edges, then it represents a flexible bar-joint framework, with at least one nontrivial motion, and a face $f$ that is not rigid during this motion. Let $x$ be a vertex on $f$ that changes its angle during the motion. Further, let $v_{1}, v_{2}, \ldots, v_{k}$ be the vertices of $f$ that are visible from $x$ in circular order. There must exist two vertices $v_{i}$ and $v_{i+1}$ for which the angle $\angle v_{i} x v_{i+1}$ changes. If edge $e=\left(v_{i}, v_{i+1}\right)$ is not in $G$ we add $e$ to $G$. If $e$ already exists, at least one distance between $x$ and $v_{i}$ or between $x$ and $v_{i+1}$ changes during the motion; we add the corresponding edge to $G$. In all cases we added an edge with endpoints in different rigid components of $G$, eliminating one degree of freedom from the space of motions of $G$. Hence, $G$ is a Laman graph or a subgraph of a Laman graph and remains $(2,3)$-sparse. Continue to add edges, until the graph is $(2,3)$-tight. See 1$]$ for an alternate proof.

Case $\mathbf{5}, k=4$. If a $(2,4)$-sparse planar graph is not bipartite, then it remains non-bipartite no matter what is added to it. Thus, it cannot be a subgraph of a maximal bipartite planar graph, which is the same thing as a $(2,4)$-tight planar graph. On the other hand, if a $(2,4)$-sparse graph is bipartite but not tight, then in any planar embedding it has a face with six or more vertices. In such a face, one can find three mutually-crossing properly colored diagonals. It is not possible for all three to already be part of the graph, because this would 


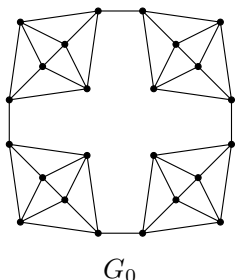

(a)

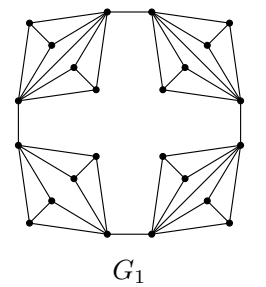

$G_{1}$

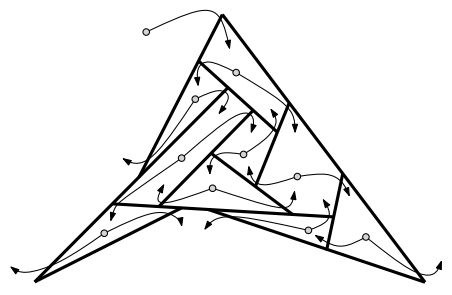

(b)

Fig. 9. (a) A plane (2,0)-tight graph $G_{0}$ and a plane $(2,1)$-tight graph $G_{1}$ whose duals are not $(2,1)$-sparse. (b) A segment contact representation of a plane (2,3)-tight graph and the corresponding orientation of the dual.

cause the graph to contain a $K_{3,3}$ minor. Therefore, one of these diagonals can be added, splitting the face while preserving bipartiteness. Repeating this operation leads to a graph in which all faces are quadrilaterals, which is $(2,4)$-tight.

\section{A.3 Duals of Sparse Graphs}

In this section we prove Lemma 2 relating the sparseness parameters of a graph and its dual.

First, let $\ell \in \mathbb{Z}$ be any integer. We shall construct a plane $(2,0)$-tight graph $G_{0}$ and a plane $(2,1)$-tight graph $G_{1}$ whose duals $G_{0}^{*}$ and $G_{1}^{*}$ contain two vertices $v$ and $w$ that are joined by $5-\ell$ parallel edges. In particular, $G_{0}^{*}$ and $G_{1}^{*}$ will not be $(2, \ell)$-sparse.

We start with a cycle $C$ of order $2(5-\ell)$ and replace every other edge $e$ of $C$ with a certain graph $H$. Let $H_{0}$ and $H_{1}$ be any plane $(2,0)$-tight graph and any plane (2,1)-tight graph, respectively, and $e_{0}, e_{1}$ be any edge in $H_{0}, H_{1}$, respectively. For $G_{0}$, we replace every other edge $e$ of $C$ with $H_{0}-e_{0}$, where the endpoints of $e_{0}$ and $e$ are identified. For $G_{1}$, we replace every other edge $e$ of $C$ with $H_{1}$, where the endpoints of $e_{1}$ and $e$ are identified, and in one such copy of $H_{1}$ we remove the edge $e_{1}$. It is easy to see that the resulting graphs $G_{0}$ and $G_{1}$ have the desired properties. We refer to Fig. 9(a) for an illustration.

Now, let $k \in\{2,3,4\}$ and $G=(V, E)$ be a planar $(2, k)$-tight graph with a fixed plane embedding. We shall show that the dual $G^{*}$ of $G$ is $(2,4-k)$-tight. By Euler's formula,

$$
\left|E\left(G^{*}\right)\right|=|E(G)|=2|V(G)|-k=2\left(\left|E\left(G^{*}\right)\right|-\left|V\left(G^{*}\right)\right|+2\right)-k
$$

and thus $\left|E\left(G^{*}\right)\right|=2\left|V\left(G^{*}\right)\right|-(4-k)$. So it remains to show that $G^{*}$ is $(2,4-k)$ sparse. We distinguish the three cases for $k$.

Case $1, k=4$. As $G$ is $(2,4)$-sparse, it is triangle-free, and as $|E(G)|=2|V(G)|-$ $4, G$ is a plane quadrangulation. In particular, all faces of $G$ are quadrangular, which means that $G^{*}$ is 4 -regular and thus $G^{*}$ is $(2,0)$-tight. 
Case 2, $k=3$. $G$ is $(2,3)$-tight, so by Lemma 11 it has a decomposition into two edge-disjoint spanning trees after doubling any edge $e$. A spanning tree $T$ in a plane graph corresponds to a spanning tree in its dual graph $G^{*}$, formed by the dual edges of all edges not in $T$. Hence, $G^{*}$ can be decomposed into two spanning trees after deleting any edge. Let $v$ be an arbitrary vertex in $G^{*}$. We delete some edge $e^{*}=(u, v)$ in $G^{*}$, fix two edge-disjoint spanning trees of $G^{*} \backslash e^{*}$, and orient each edge towards the root $v$. We end up with a 2 -orientation that meets the requirements of Lemma 12 iii), and therefore with a $(2,2)$-tight graph. Adding back $e^{*}$ results in a (2,1)-tight graph.

We remark that there is an alternative, more geometric proof for this case. As $G$ is a planar $(2,3)$-tight graph, $G$ admits a segment contact representation 8 . Every vertex $v$ of $G^{*}$, except the vertex $v_{o}$ for the outer face of $G$, corresponds to a convex polygon $P(v)$ in this representation; see Fig. 9(b). Now, we add in $G^{*}$ a loop at $v_{o}$ and obtain a 2-orientation of this graph by orienting outgoing for every $v \neq v_{o}$ the edges corresponding to the leftmost and rightmost point of $P(v)$, and the remaining two edge outgoing at $v_{o}$.

Case 3, $k=2$. As $G$ is $(2,2)$-tight, by Lemma 11, $G$ is the union of two edgedisjoint spanning trees. Since $G^{*}$ is connected, for every spanning tree $T$ of $G$, the edges of $G^{*}$ that are not crossed by any edge in $T$ form a spanning tree of $G^{*}$. Hence, $G^{*}$ is the edge-disjoint union of two spanning trees. Thus, again by Lemma 11 . $G^{*}$ is $(2,2)$-tight.

\section{B Generating Plane Laman-plus-one Graphs}

Here we prove Lemma 4 (claimed by Fekete et al. 11]), which states that every plane Laman-plus-one graph can be generated by $E_{3}$ moves starting from a $K_{4}$.

We need the following three auxiliary lemmas. The first of these lemmas is due to 11 and can be easily proved by counting.

Lemma 13. Let $G$ be a (2,3)-tight graph and let $H_{1}$ and $H_{2}$ be two subgraphs of $G$ which are both $(2,3)$-tight such that $\left|V\left(H_{1}\right) \cap V\left(H_{2}\right)\right| \geq 2$. Then $H_{1} \cup H_{2}$ is also $(2,3)$-tight.

For an edge $e$ of $G$, denote by $G / e$ the graph obtained from $G$ after contracting edge $e$. If $G$ is a plane graph, then the planar embedding of $G$ also defines a planar embedding for $G / e$. Let $G$ be plane (2,3)-tight graph and let $e$ be an edge of $G$. Then $e$ is called contractible if the graph $G / e$ is also a plane $(2,3)$-tight graph. One can see that if $G$ has a contractible edge $e$, then $G$ can be generated from $G / e$ by an $E_{3}$ move. The following lemma is due to 11 .

Lemma 14 (Fekete et al. [11]). Let $G$ be a $(2,3)$-tight graph with $|V(G)| \geq 4$ and let uvw be a triangle of $G$ such that the edge uv is not contractible. Then there is a proper $(2,3)$-tight subgraph $H$ of $G$ such that $u, v \in V(H)$ and $w \notin V(H)$. Furthermore, there is some edge $e^{\prime} \in E(H)$ which is contractible in $G$.

We also have the following lemma. 
Lemma 15. Let $G$ be a plane graph with a $K_{4}$ as a subgraph $H$ formed by four vertices $u, v, x, y$. If $G-(u, v)$ is a $(2,3)$-tight graph, then $G$ can be generated by $E_{3}$ moves starting from $H$.

Proof. Consider the four triangle faces of $H$. For each of these faces $f$, denote $U_{f}$ the set of vertices inside the face $f$. We first claim that for each of these four faces $f$, the subgraph of $G$ induced by the vertices $V(f) \cup U_{f}$ is a $(2,3)$-tight graph; where $V(f)$ denotes the vertices on the cycle $f$. Indeed, if $f$ does not contain the edge $(u, v)$ (assume $f=v x y$ since the case when $f=u x y$ is similar), then the claim follows from Lemma 6 , since both $G-(u, v)$ and $f=v x y$ are (2,3)-tight; see Fig. 10(a).

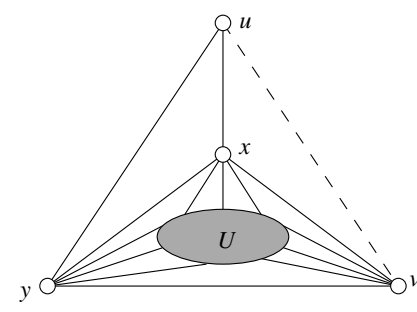

(a)

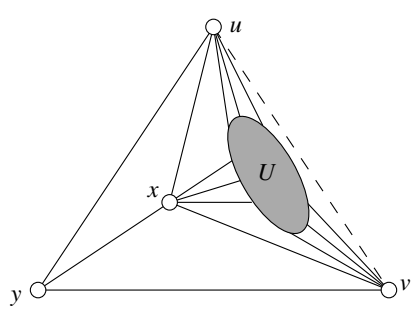

(b)

Fig. 10. Illustration for the proof of Lemma 15

Otherwise assume that $f=u v x$ (the case when $f=u v y$ is similar); see Fig. 10(b). Denote by $X$ the subgraph induced by $V(f) \cup U_{f}$. Due to Lemma 6 the subgraph of $G-(u, v)$ induced by $U_{f} \cup\{u, v, x, y\}$ is (2,3)-tight. Thus the number of edges in $X$ is $2|X|-3$. Furthermore $X-(u, v)$ is (2,3)-sparse. Therefore if $X$ is not $(2,3)$-tight, then there is subgraph $Y$ of $X$ containing $(u, v)$ such that $|E(Y)| \geq 2|V(Y)|-3$. If $x$ is not in $Y$, then add the vertex $x$ and the edges $(u, x)$ and $(v, x)$ to it. Finally delete from $Y$ the edge $(u, v)$ and add to it the vertex $y$ and the edges $(u, y),(v, y)$ and $(x, y)$. Then this graph is not $(2,3)$-sparse but is a subgraph of $G-(u, v)$, a contradiction.

We thus assume that for each face $f$ of $H$, the subgraph induced by $V(f) \cup U_{f}$ is plane $(2,3)$-tight. Therefore this graph can be obtained by $E_{3}$ moves starting with the triangle $f$ 11. Thus using these moves, one can generate $G$ starting from $H$.

We are now ready to prove Lemma 4.

Lemma 4. Every plane Laman-plus-one graph can be generated by $E_{3}$ moves starting from $K_{4}$.

Proof. Assume for a contradiction that there is a plane Laman-plus-one graph other than $K_{4}$, which cannot be generated by $E_{3}$ moves starting from $K_{4}$. Let $G$ be such a graph with the minimum number of vertices. Since $G$ is a Laman-plusone graph, there is an edge $e=(u, v)$ of $G$ such that $G-e$ is a Laman $((2,3)$-tight $)$ 
graph. We show that there is an edge $e^{\prime}=(x, y)$ such that $u, v \notin\{x, y\}$ which is contractible in $G-e$. Thus $H=\left((G-e) / e^{\prime}\right) \cup e$ is a Laman-plus-one graph with fewer vertices than $G$ and $G$ can be obtained from $H$ by an $E_{3}$ move. By the minimality of $G, H$ (and hence $G$ ) can be generated from $K_{4}$ by a sequence of $E_{3}$ moves, a contradiction.

Suppose that there is a triangle $T=x y z$ in $G-e$ such that $u, v \notin\{x, y, z\}$. If any edge of $T$ is contractible, then we are done. Otherwise by Lemma 14 there exists proper (2,3)-tight subgraphs $H_{1}, H_{2}, H_{3}$ of $G-e$ such that $x, y \in$ $V\left(H_{1}\right), z \notin V\left(H_{1}\right) ; y, z \in V\left(H_{2}\right), x \notin V\left(H_{2}\right) ;$ and $z, x \in V\left(H_{3}\right), y \notin V\left(H_{3}\right)$. Furthermore for each $i=1,2,3$, there is an edge $e_{i} \in E\left(H_{i}\right)$ which is contractible in $G-e$. By Lemma 13, $V\left(H_{1}\right) \cap V\left(H_{2}\right)=\{y\}, V\left(H_{2}\right) \cap V\left(H_{3}\right)=\{z\}$, and $V\left(H_{3}\right) \cap V\left(H_{1}\right)=\{x\}$. Therefore there is at least one $i \in\{1,2,3\}$, that contains neither $u$ nor $v$. Thus we have a desired contractile edge $e_{i}$ in $G-e$.

We thus assume that each triangle in $G$ contains either $u$ or $v$. Note that no triangle can contain both $u$ and $v$ since $G-e$ does not contain the edge $(u, v)$. Since $G-e$ is a plane $(2,3)$-tight graph, it contains at least two triangular faces. Let $u x y$ be such a triangular face (the case when we consider a triangle containing $v$ is similar). If the edge $(x, y)$ is contractible, we are done. Otherwise by Lemma 14 there is a proper (2,3)-tight subgraph $H$ of $G$ such that $x, y \in V(H)$ and $u \notin V(H)$. If $|V(H)| \geq 4$, then by 11 there is a contractible edge $e^{\prime}$ in $H$ that does not have $v$ as an endpoint. If $e^{\prime}$ is not $(x, y)$, then this gives our desired contractible edge. Otherwise since each triangle contains either $u$ or $v$, the four vertices $u, v, x, y$ form a $K_{4}$ in $G$. Thus by Lemma 15 , we can generate $G$ by $E_{3}$ moves, starting from $K_{4}$, also a contradiction.

We finish this section with a proof for Lemma 6

Lemma 6. Let $G$ be a $(2, k)$-tight graph and let $H$ be a proper $(2, k)$-tight subgraph of $G$, for $k>0$. Let $C$ and $D$ be a partition of the vertices of $G-H$ such that there is no edge between vertices in $C$ and vertices in $D$. Then both the graphs induced by the vertices of $H \cup C$ and $H \cup D$ are also $(2, k)$-tight.

Proof. Since $G$ and $H$ are both $(2, k)$-tight and $|E(C, D)|=0,|E(G)|=2|V(G)|-$ $k=2|V(H)|+2|V(C)|+2|V(D)|-k=|E(H)|+|E(C)|+|E(H, C)|+|E(D)|+$ $|E[H, D]|$, and $|E(H)|=2|V(H)|-k$. Again since $H \cup D$ is a subgraph of $G$, it is $(2, k)$-sparse, so $|E(H \cup D)|=|E(H)|+|E(D)|+|E[H, D]| \leq 2|V(H)|+2|V(D)|-k$. Thus $|E(H \cup C)|=|E(H)|+|E(C)|+|E[H, C]| \geq 2|V(H)|+2|V(C)|-k$. Since $H \cup C$ is a subgraph of $G$ and hence is $(2, k)$ sparse, this implies that it is $(2, k)$-tight. Similarly $H \cup D$ is also $(2, k)$-tight.

\section{Finding One-Sided Representation is NP-Hard}

Theorem 2. Testing whether a planar $(2,0)$-tight graph has a contact representation where the convex hull of each arc is empty is NP-complete, even for graphs of maximum degree 5 .

Proof. We first prove the theorem for plane multigraphs. By Lemma 10 it is equivalent to prove that finding a good representation of a $(2,0)$-tight plane 
graph is NP-complete. We reduce from the known NP-complete problem Positive Planar 1-in-3SAT 18 of deciding the satisfiability of a 3SAT formula with the additional restrictions that: (1) the formula contains only positive variables; (2) we seek a truth assignment such that each clause has exactly one true variable; and (3) the graph whose vertices are variables and clauses and whose edges are variable-clause incidences has a planar drawing in which all variables are arranged on a straight line and no edges cross this line.

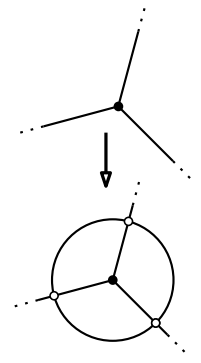

(a)

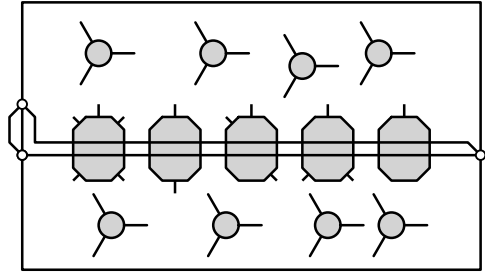

(b)

Fig. 11. (a) Removing multiple edges. (b) Piercing the variable gadgets (octagons) to get the 3-connectedness.

Our reduction uses wire, splitter, and clause gadgets (Fig. 7) based on the principle that, for a good 2-orientation at a degree-4 vertex, each incoming edge must be opposite an outgoing edge and vice versa. Each variable of the 3SAT formula is replaced by a wire gadget that is closed to a circle with doubled edges. There are two good 2-orientations of this doubled circle, the choice of which encodes the truth value of that variable. A splitter gadget with a short piece of wire propagate this signal to the clause gadgets, which represent 3SAT clauses. Due to the degree- 4 vertices, there is only one good 2-orientation for the splitter that extends a "wire signal". The degree- 3 vertex in the clause gadget verifies that exactly two of the attached wires carry a false signal. The remaining edges and vertices in the clause gadget ensure that a completion to a good 2-orientation is possible in all cases.

To finish the proof, we convert our $(2,0)$-tight multigraph into a $(2,0)$ tight simple planar graph, preserving the existence of a good 2-orientation. To accomplish this, we surround each vertex by a chain of edges and vertices (Fig. 11(a) and augment the graph to make it 3-connected (Fig. 11(b) , ensuring that our chosen combinatorial embedding is unique up to reflection. To do this we add a triangle with doubled edges that will enclose the previous construction. We route two non-parallel edges of the triangle so they cut through edges of all variable gadgets, and repair the crossings by adding new vertices at the intersections. We claim that every vertex has three vertex-disjoint paths to vertices on the boundary, which implies the 3 -connectedness. If we follow a wire in one direction we can do this in two possible vertex-disjoint ways (after the 
removal of the multiple edges). Hence, if we consider a vertex of a wire gadget we can reach the variable gadgets with three vertex-disjoint paths; two in one direction and one taking a detour via a clause gadget and another wire. From the variable gadgets we can now direct the three paths to the boundary. Again, since the variables are built from wires, we can select two paths along one "direction" and use the other direction for the third path. The argument is similar for the other vertices. Note that the augmented graph still has one multiple edge, which we can remove with the same construction. The resulting graph has maximum vertex degree 5 , in its clause gadgets.

\section{Contact Representations with Right Wedges}

Here we consider contact representation with right wedges, where the angle at the corner of each wedge is $90^{\circ}$.

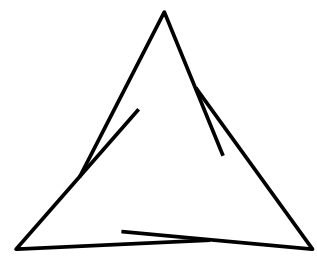

Fig. 12. A wedge-contact representation bounded by three wedges.

Lemma 16. Let $\Gamma$ be a wedge-contact representation of a (2,0)-tight graph such that the outer boundary of $\Gamma$ is bounded by exactly three wedges. Then $\Gamma$ cannot be modified to a topologically equivalent representation where all the three wedges on the outer boundary are right wedges.

Proof. Since the underlying graph is a $(2,0)$-tight graph, all the angles in the outer boundary of $\Gamma$ other than those at the three corners of the wedges are reflex angles. Thus the outer boundary of $\Gamma$ is a pseudo-triangle; see Fig. 12. If all three wedges are right angle, we have a pseudo-triangle with the summation of the three convex angles more than $180^{\circ}$; which is geometrically infeasible.

Lemma 16 immediately implies that if a plane $(2,0)$-tight graph has a triangular outer face, then it has no contact representation with right wedges. In particular, the octahedron graph is a (2,0)-tight graph and all its faces are triangles. Thus in every planar embedding of the octahedron, the outer face is a triangle, so the octahedron has no contact representation with right wedges.

\section{Acknowledgments}

This work was initiated at the workshop on Drawing Graphs and Maps with Curves held at Schloss Dagstuhl. We thank Éric Fusy for helpful comments. 
A. Schulz is supported by the German Research Foundation (DFG) under grant SCHU 2458/4-1. D. Eppstein is supported in part by NSF grant 1228639 and ONR grant N00014-08-1-1015.

\section{Additional References}

24. J. Graver, B. Servatius, and H. Servatius. Combinatorial Rigidity. Grad. Studies in Math. Amer. Math. Soc., 1993.

25. J. Roskind and R. E. Tarjan. A note on finding minimum-cost edge-disjoint spanning trees. Math. of Oper. Res., 10(4):701-708, 1985.

26. T.-S. Tay. Henneberg's method for bar and body frameworks. Structural Topology, $17,1991$. 